\title{
Der Normalsprecher
}

\section{Von Adolf Jens Koemeda}

Habe ich den Tunnel erwähnt? Nein? Entschuldigung. Ich bin immer noch nicht ganz erholt, leider, leider. Sie müssen sich bitte wehren, wenn ich alles durcheinanderbringe oder zu viel quatsche ... ja, darum möchte ich Sie sehr bitten, lieber Herr Jost, Sie dürfen mich mitten im Satz unterbrechen, ohne weiteres. Der Tunnel ... sehen Sie, ich hätte wetten können, dass ich schon gestern damit angefangen hatte. Mein Fehler!

Die Abkürzung wollte ich mir nicht entgehen lassen und den Tunnel auch nicht, deshalb fuhr ich nicht über den Ofenpass, sondern über Livigno ... bitte? Nie gehört? Also: nahe der schweizerischen Grenze, eine romantische und relativ dünn besiedelte Gegend ... Verzeihung, ich tue jetzt so, als ob es eine grobe Wissenslücke wäre, Livigno nicht zu kennen; ich weiss, den meisten ist diese Ortschaft unbekannt ... nichts für ungut. Nun, Livigno: Dort machte ich eine kurze Pause, trank Kaffee und fuhr gleich los. Zuerst am Ufer des Stausees entlang, mehrere Kilometer in der Galerie, vermutlich als Schutz vor Steinschlag und kleinen Wasserfällen. Felsen, nur Felsen, nein, kein ausgesprochen liebliches Landschaftsbild. Hatte ich in dieser Höhe eigentlich auch nicht erwartet, aber trotzdem.

Ich hatte schon früher davon gehört: lang und uralt, irgendwie originell. Sehr schmal, so schmal, dass ein Aneinander-Vorbeifahren von zwei Autos nicht möglich sei, man könne nur in einer Richtung reisen. Die Wagen auf der anderen Seite des Berges haben währenddessen Rot, hiess es, man warte zwischen fünfzehn und zwanzig Minuten. Gut, ich hatte es nicht eilig, die Strasse war jetzt leer, ich fuhr nicht über achtzig und schaute mir die Moränen und steilen Felsenwände an. Mit zwanzig Minuten Warterei kann ich mich arrangieren, dachte ich, in dieser eigenartigen Landschaft muss das gar nicht so unangenehm sein.

Glück gehabt! Keine zwanzig Minuten, nicht einmal zwei. Gleich nach der Bezahlung der Tunnelgebühr verschwand das Rot von der Ampel, und ich konnte Gas geben.
Zuerst ein Stück auf der Staumauer, dann ein paar hundert Meter zwischen den Felsen und gleich danach der Tunneleingang. Vor mir kein Auto, hinter mir keins, komisch, dachte ich, aber vielleicht besser so.

Der Tunnel von Livigno ... Sie kennen ihn nicht, sagten Sie; habe ich eigentlich auch nicht erwartet, lieber Herr Jost. Die Berge vor dem Ofenpass sind eine ziemlich menschenleere Gegend, ich habe nur zufällig am Stammtisch von diesem Tunnel gehört. Empfehlungen am Stammtisch nehme ich allerdings ernst und um offen zu sein, richtig enttäuscht haben sie mich noch nie. Also: sehr originell, teilte man mir mit, wenn ich schon in der Gegend sei, sollte ich keineswegs diese kleine Attraktion auslassen. Keine Warterei, es kam bald Grün, ich durfte gleich weiterfahren ... habe ich das nicht bereits gesagt? Ja, Glück gehabt! Hoffentlich verlässt mich mein Glück heute nicht so schnell, dachte ich und hatte zugleich Angst, dass mir gerade das passieren könnte, denn: Mehrere Tage hintereinander war das Glück auf meiner Seite gewesen, und ich befürchtete nun, dass diese sympathische Glückssträhne demnächst zu Ende sein würde. Bitte? Natürlich, ein paar Beispiele könnte ich Ihnen auf der Stelle nennen, sagen wir ... ach nein, meine Schilderung könnten Sie leicht als plumpes Prahlen missverstehen, was mir gänzlich fern liegt. Lieber gleich zum Tunnel.

Ich kontrollierte die Benzinuhr - der Tank war halb voll -, schloss das Schiebedach und schaltete die Frischluftzufuhr aus; dann verschwand ich in der felsigen Wand. $\mathrm{Na} \mathrm{ja}$, dachte ich, na ja: hinter mir niemand, vor mir niemand, so tief und ganz alleine im Schoss von Mutter Erde war ich in meinem Leben noch nie gewesen; hoffentlich geht alles gut, hoffentlich komme ich auf der anderen Seite wieder heil heraus.Wenn aber nicht, wenn etwas passieren sollte, mit mir oder mit dem Wagen, würde man es irgendwo bemerken? Gibt es Kameras, gibt es Kontrollpunkte? Ich meine, würde mich jemand retten können? Ich verlangsamte und guckte mir die schwarzen Felswände genauer an: nichts! Ich fuhr noch langsamer, fast im Schritttempo - kein Problem, hinter mir nach wie vor niemand - nein, verkehrsüberwachende Kameras konnte ich nirgends entdecken. Nanu.

Aber zu Ihrer vorherigen Frage, Herr Jost, die habe ich Ihnen gestern nur teilweise bzw. schlecht beantwortet; ich war müde, hatte viel stärkere Schmerzen als heute, und ich dachte mir, das gehe Sie gar nichts an. Gut, Sie haben recht, etwas kann ich Ihnen schon sagen.

Ich war gerade unterwegs von Meran nach Davos - ohne Begleitung ... warum? Warum alleine auf der Reise? Also, wenn Sie es sich ausgesprochen wünschen, erzähle ich ein bisschen mehr - und wenn Sie bereit wären, von sich selber ebenfalls etwas preiszugeben, wäre ich natürlich froh. Bitte: Ich stamme aus einfachen Verhältnissen, Landwirtschaft, ein kleiner Betrieb, bei uns wurde nie viel gesprochen; arbeiten und nicht reden, das war die Devise des Vaters. Meine Frau dagegen - Tochter von intellektuellen Eltern, die sich am Chiemsee niedergelassen hatten, sehr belesen und selber Hobbydichterin sprach schnell, viel und wortreich; ich fand es toll, es imponierte mir, ihre Redegewandtheit bewunderte ich seit dem ersten Augenblick. Lange, sehr lange. Aber natürlich nicht ewig.

Ihre eloquente Art begann mich auf einmal zu irritieren, zu ärgern und ... doch, doch, diese Zeit kam auch, mich wütend zu machen. Tja - warum eigentlich? Ich wusste es nicht! Meine vage Vermutung - sie wurde erst viel später bestätigt - war: weil sie sich, Annemarie hiess sie übrigens, für mich nie interessierte, von mir nichts erfahren wollte, eben - als ob ihre eigenen Überlegungen und Gedanken schon fertig wären, abgeschlossen und perfekt, keiner Ergänzung bedürftig; meine eventuellen Beiträge waren für sie also bedeutungslos. So wie ich selber.

Mit der Zeit musste ich allerdings feststellen, dass meine Frau mit dieser Haltung nicht alleine war; die meisten Menschen, glaube ich, sind heutzutage so, sie verstecken vielleicht ihr Desinteresse an den anderen gekonnter und mimen eine gewisse Offenheit und Anteilnahme geschickter und überzeugender, als es Annemarie in den letzten Jahren unserer 
Ehe getan hat. Apropos: Es soll neuerdings für dieses «Interesse-an-den-Mitmenschenmimen» einschlägige Workshops geben. Nun, zurück zu Ihrer Frage.

$\mathrm{Ja}$, ich bin geschieden, alleinstehend, bewusst und gewollt - und so weit auch zufrieden damit; nicht pausenlos, gewiss, dennoch mehrheitlich. Reicht das, lieber Herr Jost? Es gibt Momente, wo ich mir etwas anderes wünsche, gar keine Frage, sie sind aber selten, und wenn ich mir die ganze Zeit seit der Scheidung bis heute genau anschaue, sind sie, Gott sei Dank, von Monat zu Monat immer seltener geworden. Bis auf ein paar Ausnahmen, natürlich, die gibt es leider ab und zu.

Im Tunnel handelte es sich um eine solche Ausnahme, oh ja, wahrscheinlich schon in den Tagen vorher in Meran.

Ich war in einem schönen und vor allem ruhigen Hotel abgestiegen, bekam ein Zimmer mit Balkon und Bergsicht, sehr sonnig, charmante Bedienung im Speisesaal; und was die Gäste betraf: Familien noch und noch, einige junge bis sehr junge Paare, sympathische Menschen ... nur Singles, abgesehen von mir, leider keine.

Tja, in so einer Umgebung wünscht man sich eine warmherzige und aufgestellte Partnerin, ein verständliches Bedürfnis, ich glaube, Sie hätten ähnlich empfunden, lieber Herr Jost. Der Mensch ist seinem Wesen nach halt nicht Solist oder gar Dauersolist ... bitte, das ist meine persönliche Ansicht; TV und PC konnten hier etwas verändern, den natürlichen menschlichen Trieb zum Kollektiv leicht abschwächen, nicht allerdings gänzlich beseitigen. Und da sind wir Singles nicht so gut dran. Man gewöhnt sich zwar an das Alleinsein, man muss aber diesen guten, alten Hang zur Gemeinschaft und Geselligkeit bewusst bei sich bekämpfen. Ja, ich tue es, es bleibt mir nichts anderes übrig, ich tue es jedoch ungern.

Gar keine Frage: Im Tunnel hat mir eine Partnerin gefehlt, zumindest von dem Moment an, in dem der Motor zum Stillstand kam, und ich spürte, wie mich Panik und Angst in den Schwitzkasten nahmen. Sie sind sicher Autofahrer und wissen natürlich ganz gut, wie problematisch längere Tunnelfahrten sein können, und zwar nicht nur in dem dunklen und schmalen Tunnel von Livigno, sondern auch in den hellen, breiten und schön gekachelten Röhren von heute. Der Motor begann bereits nach den ersten zwei-, dreihundert Metern nach der Einfahrt leicht zu stottern, ich war irritiert, da aber die Leistung nicht nachliess, und ich das Stottern

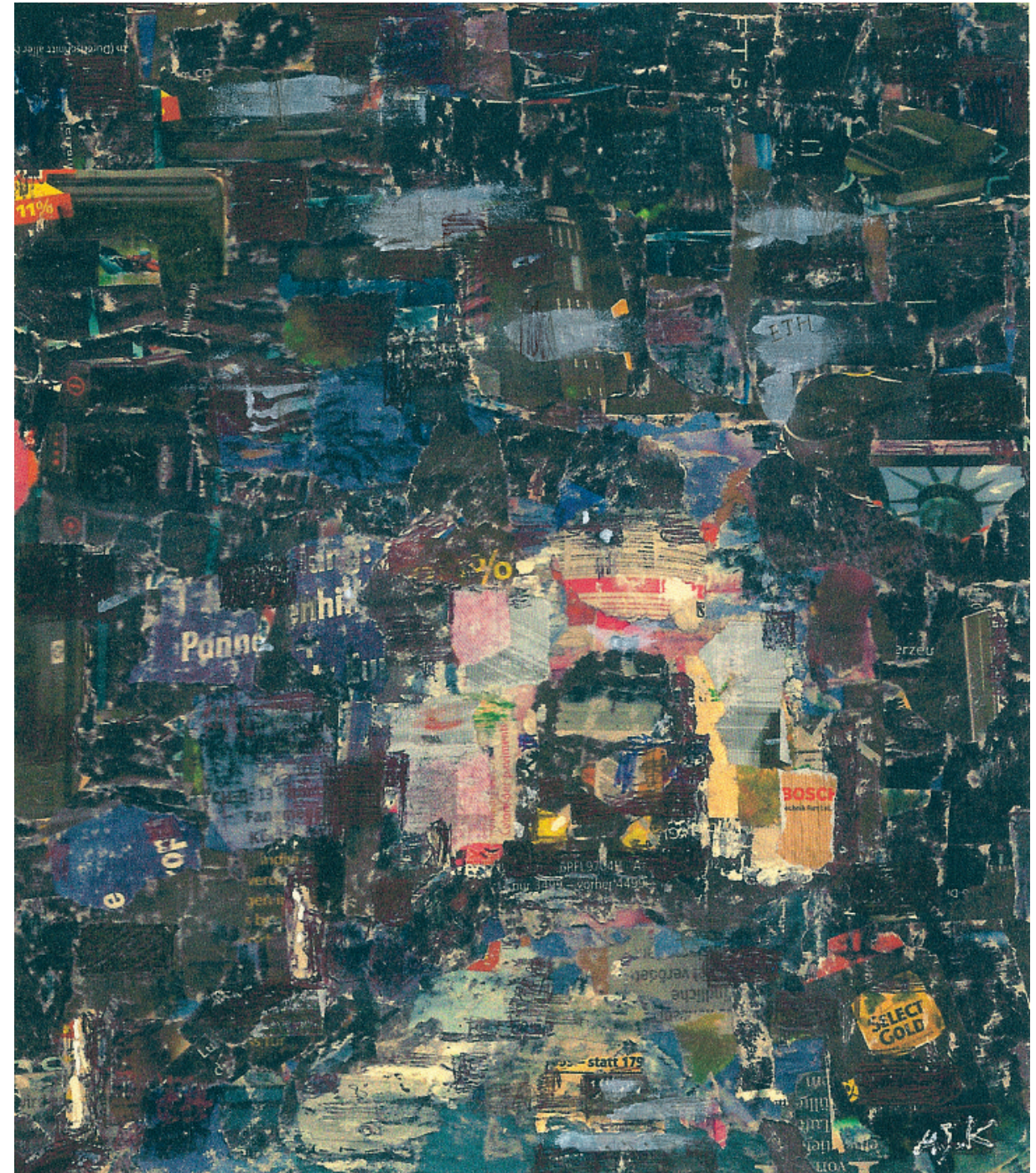

Illustration: Autor.

durch mehr Gasgeben beheben konnte, beruhigte ich mich; nicht hundertprozentig, jedoch weitgehend. Ich schaute schnell in den Rückspiegel, immer noch kein Auto hinter mir, Gott sei Dank! Nach etwa drei Minuten allerdings kein Stottern mehr, nur die Leistung war deutlich schwächer geworden, das Gasgeben half nicht weiter. Und dann plötzlich - kein Motorgeräusch! Ende, fini, Stille ... eine gespenstische Stille.

Ich schaltete sofort auf Leerlauf, liess den Wagen ausrollen und versuchte, möglichst nah an die rechte Felswand zu kommen, damit sich, wenn schon kein Auto, zumindest ein Motorrad an mir vorbei durchquetschen könnte. Der Motor lief also nicht mehr, nur das Licht brannte, Gott sei Dank!

Und jetzt? Wie weiter? Keine Ahnung!

Ich sass im Auto, wollte im ersten Augenblick singen, schimpfen, mit einem deplazierten Lachanfall rechnete ich sogar, so weit kam es aber nicht. Dummerweise tauchten plötzlich ein paar alte Fernsehbilder auf - eine Auffahrkollision, mehrere Verletzte und Tote, schrecklich -, es war im San Bernardino nein, im Sankt Gotthardtunnel, glaube ich; Bilder, die mühelos einen Henker tief erschüttern würden.

Eine Weile war ich wie gelähmt, ich wollte nur schreien, tat es auch, nach ein paar schrillen Tönen gab ich auf. Erst nach weiteren zwei, drei Minuten kam mir in den Sinn, dass ich dringend das Pannendreieck hervorholen und hinter das Fahrzeug stellen sollte, zumindest dreissig Meter entfernt. Theoretisch war es mir klar, praktisch aber ... nichts! Ich rührte mich nicht vom Fleck. Ich schimpfte weiter vor mich hin - welche Worte mir dabei einfielen, können Sie sich sicher gut vorstellen, Herr Jost. Wieder versuchte ich zu starten, der Motor knirschte und quietschte, kein normales Geräusch, 
dann wurde es still; ich hörte bloss Tropfen von den nassen Felswänden fallen ... nun, vielleicht bildete ich mir das nur ein.

Es ist doch so: Junge Dichter denken an den Tod, alte Tattergreise an junge Fräuleins; nein, so weit war es bei mir noch nicht, aber einen Partner - natürlich, am liebsten eine frische, zupackende und technisch versierte junge Partnerin - wünschte ich mir in dem Augenblick sehr, denn, was das Konkrete und Praktische anbelangt, müssen Sie wissen, habe ich zwei linke Hände.

Nach einiger Zeit machte ich einen neuen Startversuch. Trtr, trtr, trtr ... nichts! Bloss felsige Stille. Und jetzt Schluss, sagte ich mir, Ende! Sonst geht die Batterie kaputt, und du sitzt hier in totaler Dunkelheit. Eine Taschenlampe suchte ich ein paar Sekunden lang in der Türnische - eigentlich habe ich sie immer dabei -, fand allerdings nichts.

Was hätten Sie, lieber Herr Jost, in dieser äusserst schwierigen Situation gemacht? Hätten Sie, zum Beispiel, telefoniert? Wahrscheinlich. Ich auch ... wenn ich gekonnt hätte.

Ich konnte aber nicht. Erstens zu tief in der Erde, kein Empfang, zweitens Batterie zu schwach, seit drei Tagen mein Handy nicht mehr gebraucht und mich um den Akku nicht gekümmert, eigentlich normal. Ohne Telefonverbindung also, immerhin nicht in totaler Dunkelheit, Gott sei Dank!

Und endlich: Ich raffte mich auf, holte das Pannendreieck aus dem Kofferraum und lief zurück, in Richtung Süden, denn von dieser Seite drohte mir die grössere Gefahr; etwa dreissig Meter weg vom Auto blieb ich stehen. Der Boden war nass, glitschig, komisch, damit hatte ich nicht gerechnet. Ich ging noch weiter, bloss nicht stürzen, dachte ich, auf der Fahrbahn sieht dich niemand, ein Lastwagenchauffeur am allerwenigsten. Ich stellte das Pannendreieck auf den Boden, der Asphalt roch nicht nach Asphalt, sondern nach Nässe und Schimmel. Dann ging ich zurück: An meinem Wagen vorbei, weiter weiter, auf die andere Seite, etwa dreissig Meter in Fahrtrichtung; der Boden war auch hier feucht und übelriechend. Es schien mir plötzlich, dass vorne, ganz weit vor mir, kurz ein schwaches Licht zu sehen wäre, ein Motorrad ... ein Auto ... nein, es war kein Fahrzeug; wahrscheinlich nur eine Spiegelung des Lichts vom eigenen Wagen an der nassen Felswand.

Und wieder zurück.

Eine Weile stand ich grübelnd und unschlüssig vor der Tür und stieg endlich ein. Totale Stille, so total, wie ich sie noch nie erlebt hatte. Schlimm! Ich schaltete das Radio ein, hörte bloss Summen ... nach einigen Sekunden knipste ich das Gerät aus. Kein Warten auf Godot. Eher auf den Tod? Ach nein. Aber auf was eigentlich? ... Oder doch aussteigen? Nur für zwei, drei Minuten? Lieber nicht, den Luftzug draussen hatte ich vorher als kalt und unangenehm empfunden, also sitzenbleiben, düstere Gedanken und Angst nach Kräften bekämpfen, hoffen und hören ... ja, vor allem hören: $\mathrm{Ob}$ und von welcher Seite sich ein Auto näherte, ein Fahrzeug, von einem Menschen chauffiert, der entweder mein Retter oder mein Henker sein könnte.

Singen, nein, das nicht gerade, jedoch summen, ja, das musste ich, die Stille wäre für mich sonst nicht zum Aushalten gewesen. Summen und auf die Uhr schauen, das ging noch, den Sekundenzeiger beobachten, sein Zucken, seinen geduldigen Marsch um das Zifferblatt. Eins wurde mir nun schlagartig klar: In Zukunft würde ich mit meinem Auto so ungern in einen Tunnel fahren, wie ein Vegetarier an einer Schlachthofführung teilnimmt. Kennen Sie das, Herr Jost? Das Gleiche, ein Malheur im Tunnel, wahrscheinlich nicht, vielleicht aber etwas Vergleichbares? Ich selber hatte so etwas, Gott sei Dank, vorher nie erleben müssen.

Nicht schön, nein, ganz im Gegenteil. Als schrecklich empfand ich meine Lage und mit der Zeit als immer schrecklicher. Kein Sich-Arrangieren mit der Not möglich, keine Gewöhnung an die Dunkelheit - sie war gottlob nicht absolut -, keine Gewöhnung an die Stille. Ja, die Stille. Die Stille! Die Stille! Ich schrie dieses Wort, zweimal, dreimal ... ziemlich lächerlich. Ich beschloss bald, mich lieber ruhig zu verhalten.

Eigenartig! In erster Linie kam mir meine Frau in den Sinn, ich meine, meine Exfrau: Ich könne ab und zu etwas sagen, nicht die ganze Zeit so stur schweigen, giftete sie; ja, sie war oft unzufrieden mit mir, ich wehrte mich natürlich, stellte ihre Kritik in Frage, ihre häufige und meistens unbegründete Kritik. Siehst du, meldete sie dann, du kannst doch reden, wenn du willst; aber leider, wenn du den Mund aufmachst, kommt nur eine Reklamation heraus, nichts Schönes oder Aufbauendes. Auch da musste ich höflich und dennoch bestimmt widersprechen, den Zusammenhang verdeutlichen, damit sie merkte, wo die Quelle für den jüngsten Streit lag, nämlich: sicher nicht bei mir! In solchen Situationen pflegte sie jeweils laut und beleidigend $\mathrm{zu}$ werden, halt so, wie sie wirklich war: empfindlich wie eine preussische Kurfürstin. Ja, es fielen Worte, die ich, lieber Herr Jost, vor Ihnen - so lange kennen wir uns noch nicht - gar nicht wiederholen möchte.

Die Menschen kommunizieren heutzutage schlecht, sie reden oft total aneinander vorbei, es entstehen Missverständnisse und Lücken, die einmal mit Fantasievorstellungen, ein anderes Mal mit ausgesprochenen Lügen gefüllt werden. Mehr Klarheit, Herr Jost, mehr Offenheit und Liebenswürdigkeit wären notwendig - nach wie vor meine innerste Überzeugung. Ohne Sprache geht es nicht, gewiss, ich bezweifle allerdings, ob dieses Ziel mit oberflächlichem Gequatsche zu erreichen ist. Meine Frau, zum Beispiel, redet viel, das war bereits am Anfang unserer Beziehung so - ich habe es vielleicht bereits erwähnt, Entschuldigung -, ich gewöhnte mich später daran, und es störte mich ein bisschen weniger als zu Beginn; weniger, ja, das ist wahr, aber immer noch ziemlich.

Auf der anderen Seite: Es gibt keine längere Beziehung, davon gehe ich aus, die nur schön ist, die keine irritierenden oder störenden Abschnitte kennt. Am liebsten hätte ich Sie jetzt frech gefragt, ob Ihre Frauenerfahrungen diese Überlegungen widerlegen oder bestätigen ... ach nein, das frage ich Sie nicht. Nicht, dass mich das nicht interessieren würde, sondern weil Sie auf eine solche Frage wahrscheinlich gar nicht eingehen würden ... mit Recht! Sie sind eher ein schweigsamer Mensch, ein Mensch, der gut und wenig spricht und mit Vielrednern nichts anzufangen weiss.

Also, jetzt meine unmassgebliche Meinung:

Es gibt immer weniger Normalbürger auf dieser Welt. Entweder schwatzen sie zu viel, schmeissen pausenlos mit Worten um sich, hören nicht richtig zu und lassen kein normales Gespräch entstehen, oder sie schweigen hartnäckig. Die Gruppe der Normalsprecher wird von Jahr zu Jahr kleiner, jawohl, kleiner und unwichtiger.

Bitte? Ach, so meinen Sie es! Nein, nein, das sehe ich anders, lieber Herr Jost. Die Schirme sind in erster Linie schuld daran, PC- und TV-Schirme, ich bleibe dabei. Die meisten Menschen - nicht alle, das behaupte ich nicht - sind im Laufe des Tages immer häufiger allein, in ihren vier Wänden gefangen, nur ihre Schirme befinden sich in der Nähe, und mit der Zeit sind sie, die User, nicht mehr in der Lage, das Tragische, den Grad ihrer Entwicklung ins Abseits, richtig wahrzunehmen. Sie leben halt in ihrer digitalen Welt, und die andere, die reale, interessiert sie von Monat zu Monat weniger; und wenn doch ein bisschen, dann wird sie falsch wahr- 
genommen, ziemlich verzerrt. Meine Frau, zum Beispiel: Ich habe mich, behauptete sie nach der Scheidung, verändert, ich rede jetzt viel, zuweilen viel zu viel; aus mir sei ein Plapperer geworden, bei einem Mann eher ungewöhnlich.

Nach der ersten Attacke dieser Art wusste ich nicht, ob ich weinen oder lauthals lachen sollte ... oder verzweifelt den Kopf schütteln. Ich sagte nur - was, was erzählst du da! Du projizierst hier etwas, was mit mir nichts zu tun hat! Sie schmunzelte überheblich-besserwisserisch, schüttelte den Kopf und schwieg. Und auf einmal redete sie und redete, ich kam gar nicht zu Wort. Ja, ich muss es wiederholen, lieber Herr Jost. Es gibt vor allem Vielredner und Totalschweiger, das ist die heutige Krux; unsere Welt wird, was die Sprache betrifft, allmählich schwarz-weiss: weniger Zwischentöne, Differenzierungen, wenig Glanz gebende Feinheiten; plump wird alles, oft sogar grob. Eben: entweder - oder. Ich hoffe, Sie verstehen mich ... oh ja, Sie nicken, das freut mich, das freut mich sehr.
Schweigen, dann wieder summen, den hüpfenden Sekundenzeiger meiner Uhr beobachten, das waren die einzigen Beschäftigungen auch in den folgenden Minuten im Auto. Und frieren, ja, ja, das kam immer mehr dazu. Also lieber hinaus, Bewegung, die Beine vertreten, mit beiden Händen Oberschenkel und Oberarme beklopfen, vielleicht - nur probeweise - leise singen.

Ich stieg aus, wäre beinahe ausgerutscht ... und sofort war die Angst wieder da: Kann ein normales Auto - geschweige denn ein Laster - bei dieser Nässe überhaupt bremsen? Wie lange wäre dann die Bremsstrecke? Sind die etwa dreissig Meter, die meinen Wagen vom Pannendreieck trennen, ausreichend?

Ich lief zurück. Relativ schnell. Und es schien mir plötzlich, dass die Strasse eine milde Steigung aufwies. Ich verlangsamte, machte ein paar Schritte rückwärts, ja, zu meinem Pannendreieck ging es bergauf, nicht sehr ausgeprägt zwar und dennoch spürbar; offensichtlich hatte der Wagen erst hinter der Tunnelmitte gestreikt, das Gefälle wies nach Norden, die Passhöhe des Tunnels war also überwunden. Jetzt erschrak ich: In diesem Fall drohte mir die grössere Gefahr von der nördlichen Seite, von der Seite her, wo auf dem Boden nichts stand - keine Warnlampe, kein Pannendreieck, gar nichts.

Ich begann zu rennen und wäre beinahe wieder ausgerutscht! Lieber schnelle und kurze Schritte. Das Pannendreieck holen und gleich zurück; an meinem Wagen vorbei und vorwärts, auf die andere Seite, Richtung Tunnelausgang-Nord. Fünfzig Meter, nein, mehr als fünfzig, denn bald kommt die lang gezogene Rechtskurve; erst dann kann der Fahrer mein Signal überhaupt sehen.

Ich stellte das Pannendreieck auf den Boden. Der Durchzug beunruhigte mich. Bleibt es so? Oder würde der Wind demnächst eher zunehmen? Würde mein Dreieck womöglich kippen und für den herannahenden Autolenker unsichtbar sein?

Fortsetzung in der nächsten Ausgabe der Schweizerischen Ärztezeitung 